\title{
Les maladies du génome mitochondrial
}

Les affections du génome mitochondrial (ADNmt) ont été décrites pour la première fois en 1988. Leur connaissance a progressé rapidement [1, 2]. Sur le plan clinique, elles provoquent surtout des encéphalomyopathies que l'on divise en trois groupes principaux : ophtalmoplégie externe progressive (PEO), épilepsies myocloniques avec fibres musculaires en lambeaux (MERRF, myoclonus epilepsy with ragged-red fibers); myopathie, encéphalopathie, acidose lactique avec crises (MELAS, mitochondrial myopathy, encephalopathy, lactic acidosis and stroke-like episodes). Sont venues s'y joindre la maladie de Leber, avec son atrophie optique, et, débordant le cadre neuromusculaire, des affections comme la maladie de Pearson, qui atteint l'hématopoïèse et le pancréas [1]. Dès le début, on a constaté deux phénomènes fondamentaux : l'existence de délétions dans l'ADNmt, atteignant plusieurs milliers de paires de bases, en règle uniques chez un sujet donné mais variables d'un malade à l'autre, avec toutefois une délétion plus fréquente que les autres, dite délétion commune ; et l'hétéroplasmie, c'est-à-dire la coexistence de deux types d'ADNmt, l'un normal, l'autre délété ; l'importance relative des deux types, ainsi que la généralisation de l'hétéroplasmie à tous les tissus ou, au contraire, sa restriction à un ou deux d'entre eux, jouent certainement un rôle dans la nature et la gravité du syndrome clinique. Lorsque ces affections sont héréditaires, elles reconnaissent le plus souvent une origine maternelle, ce à quoi on pouvait s'attendre, puisque les mitochondries proviennent uniquement de la mère ; mais, dans la plupart des cas, elles sont sporadiques, et la mutation est apparue de novo.

Les progrès récents ont porté essentiellement sur trois domaines: la compréhension du mécanisme des ponctuelles et non plus seulement de délétions, en particulier dans des gènes codant pour des $A R N$ de transfert; le rôle enfin du noyau, à coup sûr dans certaines formes familiales à hérédité mendélienne, mais peut-être aussi dans toutes, ou du moins la majorité des délétions de l'ADN mitochondrial.

Le mécanisme de production des délétions a été élucidé, au moins partiellement, grâce à l'analyse des séquences de l'ADNmt et des limites des délétions dans de nombreux cas [3-5]. Cet ADN comporte en effet des séquences répétées directes ; une recherche sur ordinateur a révélé 198 séquences dupliquées d'au moins dix bases consécutives. Dans le travail le plus complet [6], qui porte sur 30 malades, on trouve deux types opposés : dans les deux tiers des cas, la délétion est flanquée par deux répétitions directes parfaites, normalement situées aux deux bornes de la délétion, et dont une seule est retrouvée dans la séquence délétée ; chez 12 sujets, on trouvait la "délétion commune ", point chaud de délétion, qui compte 4977 paires de bases, entre les nucléotides 8482 et 13460 ; on la trouve habituellement dans les $\mathrm{PEO}$, mais un des malades atteints de maladie de Pearson et décrit dans [1] la présentait aussi. En revanche, chez un tiers des sujets, on n'a pas trouvé ces répétitions aux bornes des délétions. La délétion commune a même été détectée chez des sujets normaux : dans l'organisme adulte ou sénescent, on en compte environ une molécule pour 1000 normales - contre moins de une sur 1000000 chez le fœtus [7]. Un modèle plausible de recombinaisonglissement a été proposé lorsqu'existent des répétitions [3]. Une délétion de novo ne pourrait se produire que quand les deux brins d'ADNmt sont séparés, donc essentiellement au cours de la réplication. A cette occasion, une courbure anormale d'un des brins peut mettre en contact deux répétitions entre lesquelles une recombinaison peut se produire (figure 1). Il est à noter que ce mécanisme, qui aboutit à la formation de molécules d'ADNmt de tailles différentes, existe à l'état normal dans des espèces animales poïkilothermes comme certains lézards, dont le génome mitochondrial est moins compact que celui des mammifères.

Les mutations ponctuelles. C'est Wallace et al. [8] qui ont découvert en 1988 la première mutation ponctuelle dans l'ADNmt. Une mutation homoplasmique (portant donc sur toutes les molécules) du nucléotide 11778 remplace une arginine par une histidine au codon 340 de la NADH déshydrogénase chez la plupart des malades atteints de l'atrophie optique à hérédité maternelle appelée maladie de Leber.

L'année 1990 a vu la révélation de mutations, dans les gènes de plusieurs ARN de transfert, reconnues responsables de deux des principaux syndromes mitochondriaux. Des déficits en certains ARNt existent nécessairement lors des délétions, puisque leur gène est éventuellement inclus dans la partie délétée; leur absence entraîne, dans les mitochondries correspondantes, un défaut de synthèse protéique général. Cette observation a conduit à examiner de plus près les gènes des $\mathrm{ARNt}$, notamment dans des maladies à hérédité maternelle et sans délétion décelable.

Chez des sujets atteints de MERRF, Shoffner et al. [9] ont ainsi découvert une mutation unique de la paire de nucléotides en position 8344, créant une transition $A \rightarrow G$ dans le gène de l'ARNt de la lysine. Ce nucléotide est conservé au long de l'évolution et la mutation modifie une des boucles de l'ARNt. Elle crée un site de restriction pour l'enzyme CvijJ, permettant aisément son diagnostic. Cette observation a déjà été confirmée par plusieurs laboratoires. 


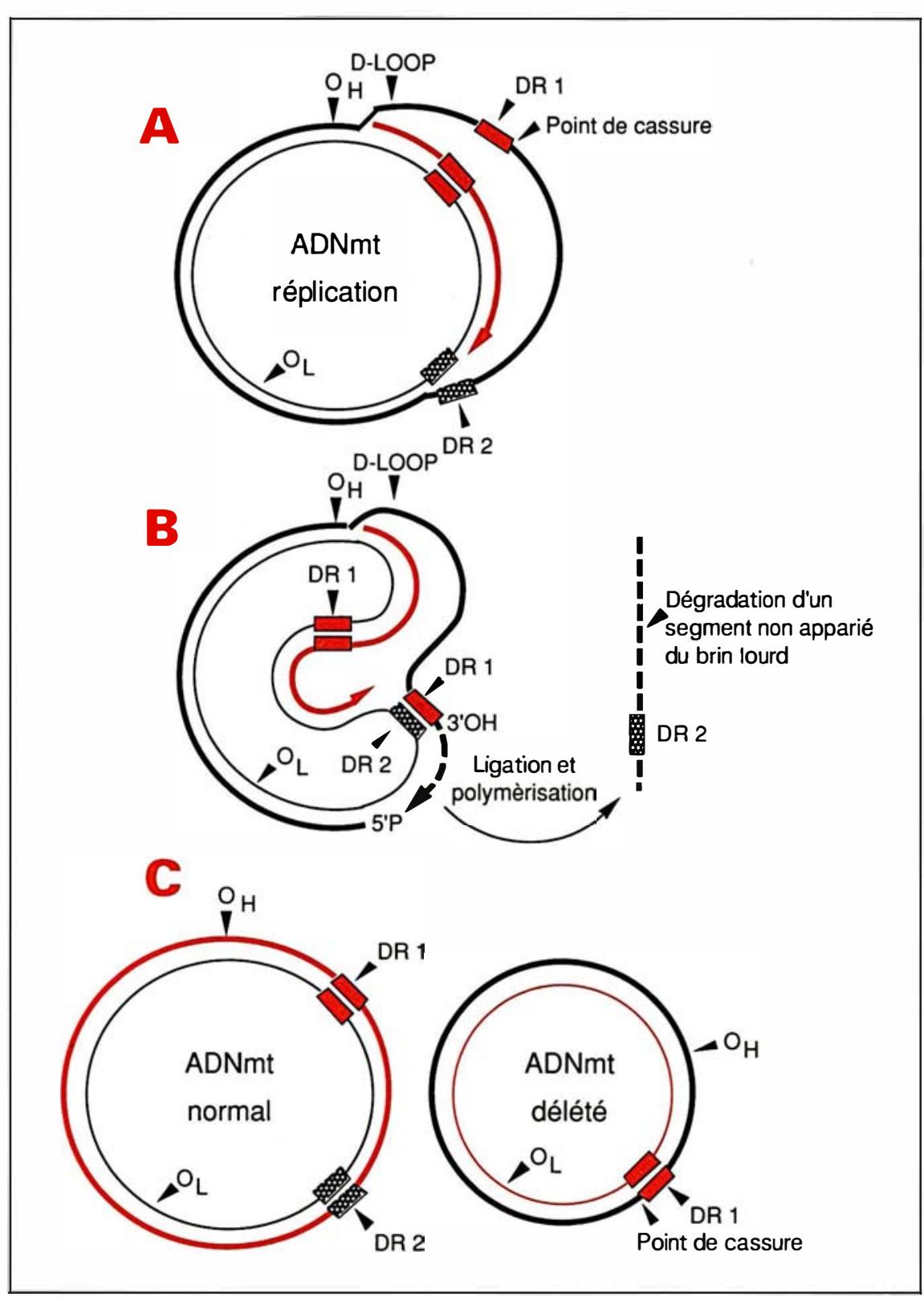

Figure 1. Modèle hypothétique de délétion par glissement-réplication. $D R$ : répétition directe. Ligne épaisse : brin parental lourd; ligne fine : brin parental léger; en rouge: brin répliqué. $O_{H}$ : origine du brin lourd; $O_{1}$ : origine du brin léger; D-LOOP: boucle de développement. (Modifiée d'après [5].)

Goto et al., au Japon [10], ont découvert chez un sujet atteint de MELAS une mutation unique du nucléotide 3243 dans un gène de l'ARNt de la leucine, correspondant à la première base de la boucle dihydro-uridine, strictement invariable de l'oursin à l'homme. Cette mutation crée un site de scission pour l'enzyme ApaI, rendant aisée sa détection. Sur 31 malades indépendants, 26 la démon$m / s n^{\circ} 2$, vol. 7 , février 91 sance des protéines dont la synthèse est gouvernée par ces ARN pourrait constituer un jalon important.

L'influence du noyau sur les altérations de l'ADNmt. En 1989 est parue l'observation surprenante (voir $\mathrm{m} / \mathrm{s} n^{\circ} 7$, vol. 5, p. 515) d'une famille chez laquelle des délétions d'ADN étaient transmises selon un mode mendélien autosomique dominant. Cette même équipe italienne vient de publier un article [11] étendant les constatations initiales à 3 familles, dont une suivie sur trois générations, tandis qu'une autre famille, présentant apparemment une hérédité autosomique récessive, était décrite au Japon [12]. Contrairement à ce que l'on trouve dans les cas sporadiques, on observe, dans une même famille, et aussi chez un même malade, des délétions multiples, bien que variables au sein de la même famille. Par exemple, chez les deux frères suivis par Yusaki et al. [12], on a mis en évidence au moins 12 délétions différentes, allant de 490 à 4600 paires de bases. Des constatations analogues ont été faites par Zeviani et al. [11]. Certaines régions du génome sont frappées de façon préférentielle, d'autres restent presque indemnes. La zone de la boucle D ou boucle de déplacement, qui contient les promoteurs et l'origine du brin lourd, n'est pas touchée. Le mécanisme de formation des délétions est le même que celui qui a été décrit précédemment : dans la famille japonaise, toutes les délétions sont flanquées par des répétitions directes, dont certaines, il est vrai, sont très courtes (4 à $12 \mathrm{pb}$ ). Des conclusions analogues dérivent des données de Zeviani et al.

Aucune indication précise n'existe encore, en revanche, concernant le problème essentiel, c'est-à-dire la nature du ou des gènes nucléaires responsables de ces maladies. Il est probable que manque ou est modifiée une des protéines chargées de lier l'ADNmt et d'en empêcher le glissement lors de sa réplication. L'identification de telles protéines s'annonce comme une tâche ardue. Toutefois, déjà plusieurs protéines candidates sont en cours d'étude. Quand elle aboutira, cette recherche démontrera peut-être que, dans la plupart sinon tous les cas de délétions de 
l'ADNmt, intervient un défaut d'interaction avec des protéines de liaison d'origine nucléaire.

\section{Jean-Claude Dreyfus}

\section{RÉFÉRENCES}

1. Rötig A, Bonnefont JP, Cormier V,et al. Les remaniements du génome mitochondrial dans les déficits énergétiques de l'enfant : de nouvelles maladies de système. médecine/sciences 1989 ; 5 : 459-71.

2. Nelson I, Degoul F, Marsac C, Ponsot $G$, Lestienne P. Des délćtions de l'ADN mitochondrial dans le syndrome de KearnsSayre et d'autres myopathies avec ophtalmoplćgie externe progressive. médecine/sciences $1989 ; 5: 472-9$

3. Shoffner JM, Lott MT, Voljavec AS, Soueidan SA, Costigan DA, Wallace DC. Spontaneous Kearns-Sayre/chronic external ophtalmoplegia plus syndrome associated with a mitochondrial DNA deletion : a slipreplication model and metabolic therapy. Proc Nall Acad Sci USA 1989 ; 86 : 7952-6. 4. Johns DR, Rutledge SL, Stine OC, Hurko O. Directly repeated sequences associated with pathogenic mitochondrial DNA deletions. Proc Nall Acad Sci USA 1989 ; 86 : 8059-62.

5. Schon EA, Rizzuto R, Moraes CT, Nakase H, Zeviani M, DiMauro S. A direct repeat is a hotspot for large-scale deletion of human mitochondrial DNA. Science 1989 ; $244: 346-9$.

6. Mita S, Rizzuto R, Moraes CT, el al. Recombination via flanking direct repeats is a major cause of large-scale deletions of human mitochondrial DNA. Nucleic Acids Res $1990 ; 18$ : 561-7.

7. Cortopassi GA, Arnheim N. Detection of a specific mitochondrial DNA deletion in tissues of older humans. Nucleic Acids Res $1990 ; 18: 6927-33$

8. Wallace DC, Singh G, Lott MT, el al. Mitochondrial DNA mutation associated with Leber's hereditary optic neuropathy. Science $1988 ; 242$ : 1427-30.

9. Shoffner JM, Lott MT, Lezza AMS, Seibel P, Ballinger SW, Wallace DC. Myoclonic epilepsy and ragged-red fiber disease (MERRF) is associated with a mitochondrial DNA tRNA lys mutation. Cell 1990 ; 61: $931-7$.

10. Goto Y, Nonaka I Horai S. A mutation in the tRNA Leu(UVR) gene associated with the MELAS subgroup of mitochondrial encephalomyopathies. Nalure 1990 ; 348 : 651-3.

11. Zeviani M, Bresolin N, Gellera C, el al. Nucleus-driven multiple large-scale deletions of the human mitochondrial genome : a new autosomal dominant disease. $\mathrm{Am} J$ Hum Genel 1990 ; 47 : 904-14.

12. Yusaki M, Ohkoshi N, Kanazawa I, Kagawa T, Ohta S. Multiple deletions in mitochondrial DNA at direct repeats of nonD-loop regions in cases of familial mitochondrial myopathy. Biochem Biophys Res Commun 1989 ; 164 : 1352-7. 